tions. Des mesures sur le magnétisme terrestre furent faites sur une bonne partie de l'itinéraire; des récoltes de préhistoire et d'échantillons de paléosols, de sables et de charbons permettront de résoudre certaines questions concernant le passé lointain de cette partie du Sahara et un herbier de 300 numéros fut rapporté.

Beaucoup reste encore à faire dans ce secteur peu accessible, au point de vue scientifique, mais cette mission aura permis, aux préhistoriens en particulier, de reconnaître les principaux points qui méritent une étude ultérieure plus détaillée.

Cette collaboration de l'industrie et de la science - chose nouvelle au Sahara - s'est donc avérée des plus fécondes et il est à souhaiter qu'elle se poursuive dans l'avenir.

(Communication du Dr. R. Mauny)

\title{
Columbia University: West African Languages Survey
}

The Ford Foundation has recently granted to Columbia University approximately $\$ 180,000$ for a 'West African Language Survey', a three-year project sponsored by a number of educational and research institutions in West Africa.

The objects of the project include substantive research in the indigenous languages of West Africa, the strengthening of West African institutions in the areas of general and African linguistics, and the training of Africans in linguistic research.

The programme includes field fellowships and grants-in-aid for linguistic research, and funds for the publication of linguistic materials concerning the West African area. The Director of the project is Professor Joseph H. Greenberg of Columbia University.

\section{(Communicated by Professor Joseph H. Greenberg)}

\section{Symposium on African Affairs at Lafayette College}

A symposium on the relevance to modern Africa of the revolutionary experience of the United States was held at Lafayette College, Easton, Pennsylvania, on ro and I I March. The principal speakers were Professor Gwendolen Carter, Mr. H. V. H. Sekyi, of the Ghana Embassy, Dr. Harry Rudin, Dr. Elliot Skinner, Dr. Eduardo Mondlane, Dr. Emory Ross, and Dr. Broadus Mitchell.

\section{L'Association Internationale de Droit Africain: la Section Belge}

CRÉÉE à Bruxelles dans les derniers mois de l'année I959, la section belge de cette Association a, depuis lors, poursuivi la tâche qui était la sienne: préparer la coopération avec les États d'Afrique et plus particulièrement le Congo belge en vue d'aider ces pays à résoudre les problèmes qui se présentent à eux dans le domaine du droit. Dans l'immédiat, la section belge s'était assignée deux objectifs: d'une part la création de sections indépendantes de l'Association en Afrique belge, d'autre part la préparation, en Belgique, d'un organe permanent à même d'assurer de manière efficace les tâches de coopération dans le domaine du droit. Il est déjà permis d'espérer la création prochaine de nouvelles sections à Coquilhatville, Élisabethville et Léopoldville ainsi qu'au Ruanda-Urundi. D'autre part la section belge multiplie les contacts afin que des sections soient aussi établies dans les autres provinces du Congo.

Outre ce premier objectif, la section belge souhaitait pouvoir constituer en Belgique un organisme permanent qui aurait pour tâche de centraliser, afin d'y répondre le plus rapidement possible, les demandes éventuelles de coopération émanant d'États africains. En outre, cet organisme devrait constituer, pour la première fois en Belgique, un véritable centre d'études du droit africain. Des contacts ont été, d'ores et déjà, pris avec le Ministère de l'Instruction Publique et on peut dire que la constitution de ce centre est en bonne voie. 
Le Comité de la section belge a fixé le montant des cotisations pour les membres ordinaires à 150 fr.; la section admet en outre des membres protecteurs dont la cotisation est laissée à l'appréciation et à la générosité de ces membres. Toutes les cotisations peuvent être versées au C.C.P. no 720588 de la section belge de l'Association Internationale de Droit Africain.

(Communication du Dr. J. Vanderlinden)

\section{L'Institut de Sociologie Solvay: Création du Centre d'Histoire et d'Ethnologie Juridiques}

L'Instrutut de Sociologie Solvay vient de créer un Centre d'Histoire et d'Ethnologie juridiques placé sous la direction du Professeur J. Gilissen. Le but de la nouvelle institution est de promouvoir les recherches sur l'évolution générale du droit et des institutions, depuis l'antiquité jusqu'à nos jours, ainsi que sur les droits coutumiers des peuples primitifs.

Les activités du Centre consistent d'une part dans l'élaboration d'instruments de travail tels que bibliographie, répertoires des sources juridiques, etc., d'autre part dans l'organisation de colloques et de séminaires sur les divers aspects de l'évolution du droit. Il est dès à présent envisagé d'organiser un colloque international sur les méthodes de rédaction des coutumes, en vue de comparer les méthodes adoptées jadis en Europe et en Amérique et actuellement en Afrique et en Asie. Enfin, par des missions de recherche en Afrique Centrale, l'étude des transformations subies par les droits coutumiers au contact du droit européen pourra être systématiquement poursuivie.

\section{Economic Study in Ruanda}

A PROJECT to study a small community in Eastern Ruanda, with emphasis on its economy and its relation to world market economy, is being undertaken by M. Pierre Bettez Gravel under the auspices of the Department of Anthropology of the University of Michigan. Particular attention will be paid to the subsistence role of livestock in the community and to the mechanisms regulating this role.

\section{(Communicated by M. Pierre Bettez Gravel)}

\section{Séminaire en Sciences Humaines de l'IRS AC}

Du 3 à 6 février inclus s'est tenu à Lwiro (Bukavu) le séminaire annuel en sciences humaines de l'IRSAC. Les chercheurs associés de l'Institut y ont présenté les communications suivantes: M. E. Van de Walle, Problèmes de méthode d'étude démographique du RuandaUrundi; M. F. Crine, La Parenté dans l'organisation politique lunda: le Prof. A. Merriam, Preliminary Study of the Political Organization of a Bala Village; Dr. J. Vansina, Dieu sur la terre: Le Roi et la distribution du pouvoir chez les Kuba; M. A. Doutreloux, Le Rôle social et politique du nkisi au Mayumbe; M.l'Abbé A. Kagame, La Cour du Rwanda ancien; le Prof. J. Jacobs, La Nouvelle Orthographe tetela-kusu; le Prof. H. Codère, Recherches en cours au Ruanda; M. M. d'Hertefelt, Acculturation politique au Ruanda; le Prof. A. Coupez, Organisation formelle du dictionnaire rwanda en préparation; le R.P. G. Hulstaert, Règle et exception en Mongo; le R.P. F. Van Moorsel, L'Archéologie de la plaine de Léopoldville; le Prof. L. Baeck, État des recherches économiques au Congo Belge et au Ruanda-Urundi; le Prof. D. Biebuyck, État des recherches en anthropologie culturelle au Congo Belge et au Ruanda-Urundi. Quoique le séminaire n'ait pas eu de thème central il a été centré en pratique autour des problèmes du Ruanda-Urundi et des problèmes de structures politiques. 